\title{
Dynamics of risers for earthquake resistant designs
}

\author{
Duan Menglan ${ }^{1,2 *}$, Wang Yi ${ }^{1}$, Yue Zhiyong ${ }^{3}$, Estefen Segen ${ }^{4}$ \\ and Yang Xiaogang ${ }^{5}$
}

\author{
${ }^{1}$ School of Naval Architecture and Marine Engineering, Dalian University of Technology, Dalian, Liaoning 116024, China \\ ${ }^{2}$ Offshore Oil/Gas Research Center, China University of Petroleum, Beijing 102249, China \\ ${ }^{3}$ Department of Mechanics and Engineering, Peking University, Beijing 100080, China \\ ${ }^{4}$ COPPE, Federal University of Rio de Janeiro, P.O.Box 68508 RJ 21945-970, Brazil \\ ${ }^{5}$ Design Division, CNOOC Engineering, Tanggu, Tianjin 300452, China
}

(C) China University of Petroleum (Beijing) and Springer-Verlag Berlin Heidelberg 2010

\begin{abstract}
It is well known that no criterion about seismic design for risers is available, and relevant research has not been reported. A comprehensive study of riser dynamics during earthquakes is performed in this paper. A dynamic model for seismic analysis of risers is developed in accordance with the working environment of the risers and the influence of inertia force of the pipelines. The dynamic equations for the developed model are derived and resolved on the basis of the energy theory of beams. Numerical simulation for an engineering project in the Bohai Oil Field, China shows that the fundamental frequency of the riser plays the major role in the seismic responses, and for platforms in shallow water in Bohai Bay, the risers demonstrate a much lower stress response due to prominent differences between the riser frequency and the earthquake wave frequency. The presented model and its corresponding method for seismic analysis are practical and important for riser design resistant to earthquake waves.
\end{abstract}

Key words: Subsea pipeline, riser, seismic design, dynamic response, earthquake wave, Hamilton theory

\section{Introduction}

There are plenty of offshore oil/gas resources in China, and large numbers of subsea pipelines need to be built in the near future. However, these oil/gas resources are in the circum-Pacific seismic belt, the most active seismic zone in the world. According to incomplete statistics, $85 \%$ of the total amount of the earthquakes in China occurs in the ocean (Sun et al, 2003). Research on subsea pipelines in seismic conditions is one of the academic and engineering attractions in offshore engineering. Romagnoli and Varvelli (1988) applied the stochastic method coupled with FEM modeling techniques to predicting possible pipeline failures in earthquakes. Matsubara and Hoshiya (2000) investigated buried pipelines for seismic design by taking the soil spring into account. They analyzed the soil spring constant in the longitudinal direction of a buried pipeline, which is one of key parameters for the seismic design of subsea pipelines, and found that the spring constant depends mainly on the shear modulus of the soil deposits and the ratio of the radius of zero displacement over the radius of a buried pipeline structure. Ogawa and Koike (2001) presented their research on structural design of buried pipelines for severe earthquakes

*Corresponding author. email: mlduan@cup.edu.cn

Received August 14, 2009 where the soil spring of the buried pipelines was also focused for seismic design. Kershenbaum et al (2000) studied the behavior of unburied subsea pipelines under seismic faults, showing that seismic faults have less effect on the straight than snaked pipelines, and an order increase in seismic magnitude causes very little change in unburied pipeline bending and total longitudinal stresses. The results are in favor of reduction in cost for subsea pipeline construction and operation. Other studies of the behavior of subsea pipelines all pay attention to some factors affecting the seismic designs of subsea pipelines, and got some better results (Bruschi et al, 1993; Tura et al, 1994; Paulin et al, 1997). However, these results have not been integrated into a seismic design code for subsea pipelines. Bruschi et al (1996) pointed out such problems that the international classification societies do not have seismic design rules for subsea pipelines. China Classification Society (CCS, 1992) required that the seismic design be carried out for subsea pipelines in seismic areas, but did not provide any details on how to do. Recently, much more work (Duan and Sun, 2004; Sun et al, 2004; 2005; 2006) has been conducted for seismic design of pipelines supported by China National Offshore Oil Corporation. They carried out a comprehensive study of soil-pipeline interaction in earthquakes, the ultimate stress calculations and their applications. Their results show that the ultimate shearing strength of the soil surrounding subsea pipelines decreases 
greatly because of its submerged unit weight and nearly saturated water content, and the elastic constraint zone of the soil acting on the pipelines decreases sharply, which will force the constrained soil into a plastic slippage state. These findings indicate that the current seismic design method based on elastic constraint state cannot be applied simply to the design of subsea pipelines. A criterion is developed to predict the constraint state between soil and the pipelines, on which engineering calculations for several subsea pipeline projects from CNOOC are based. Their research demonstrates that the elastic constraint of the soil on the pipeline is quite small and the plastic slippage of the soil takes place soon after the elastic state. It is strongly recommended that the constraint state of the soil on the subsea pipeline be determined by the developed criterion before calculating the seismic stress of pipelines, and the seismic stress be calculated based on the plastic slippage theory if plastic constraint state of the soil is predicted. It is also interesting to find that an increase in the outer diameter of the pipeline does not have significant effect on the decrease in the stress because of the increase in the constraint of the soil on the pipeline, and, increasing wall thickness of the pipeline and decreasing buried depth are two effective measures for decreasing the ultimate seismic stress.

Risers form an important part of subsea pipelines. However, no results have been reported for riser seismic design (Xie et al, 2004; CCS, 1992; DNV, 2000; 2001), except those from Yue et al (2006). They evaluated the riser strength against earthquake waves by establishing mechanical models. A simple formula including a dynamic coefficient is given to calculate the maximum stress of the risers based on the method of maximum earthquake acceleration.

In this paper, a dynamic model for seismic response analysis of risers is developed in accordance with the working environment of the risers and the influence of inertia force of the pipelines. The energy theory of beams is applied to the derivation of the dynamic equations for the developed model. A lot of numerical simulation is performed for an engineering project in the Bohai Oil Field. The results show that the fundamental frequency of the riser plays the major role in the seismic responses. It can be seen for platforms in shallow waters in the Bohai Bay, the risers demonstrate a much lower stress responses due to prominent difference between the riser frequency and the earthquake wave frequency, i.e., no resonant vibration will be expected in this case. However, for waters deeper than $100 \mathrm{~m}$, the seismic stress responses of risers can not be ignored. The presented model and its corresponding method for seismic analysis can be applied to riser design against earthquake waves.

\section{Dynamic model for risers and derivation of the dynamic equations}

The mechanical model of the pipeline system from the oil platform to the buried pipelines is presented in Fig. 1, where the part $J H$ is the riser. The mechanical model of the riser is shown in Fig. 2, where the riser is fixed at the support $J$, and also at the support $H$ in the $z$ direction and the rotation is restricted around $y$ direction. The riser is restricted at the support $H$ in the $x$ direction by simplified springs.

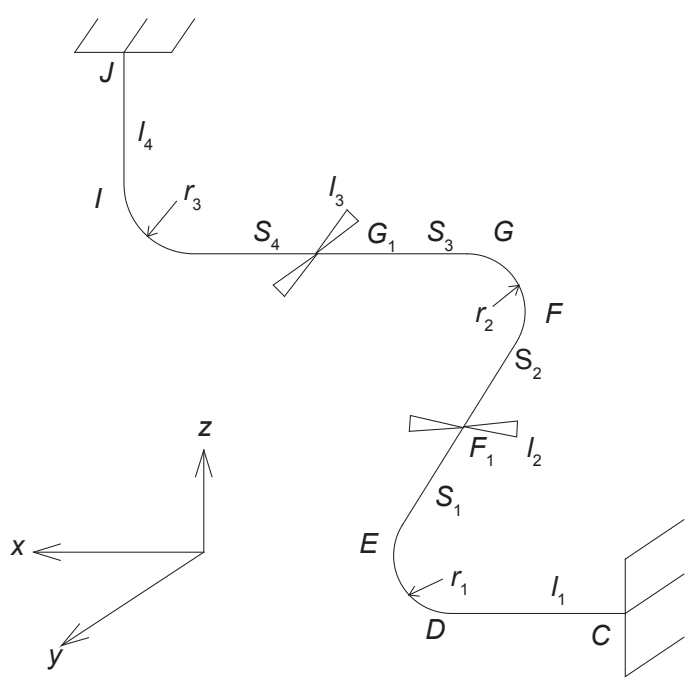

Fig. 1 The pipeline system from oil platform to buried pipelines

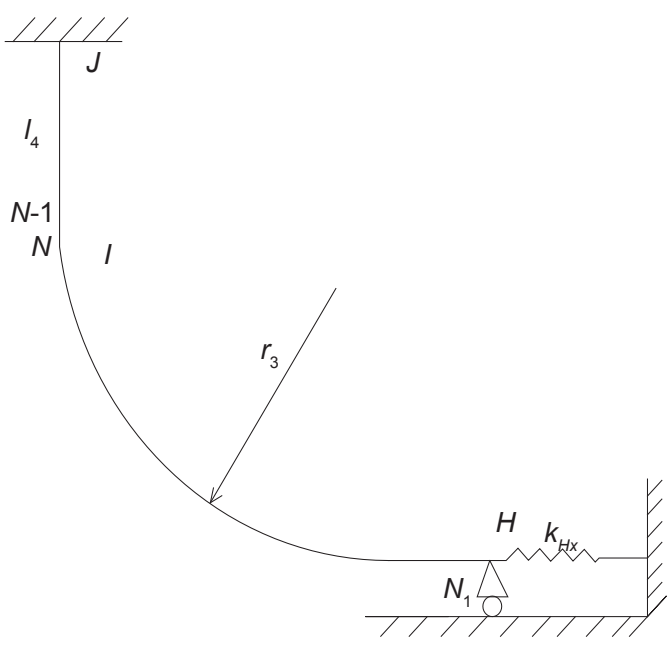

Fig. 2 Simplified mechanical model of part $J H$ of the riser

For convenience, the riser $J H$ is separated into to a straight part $J I$ and a curved part $I H$ as modeled respectively in Fig. 3 and Fig. 4.

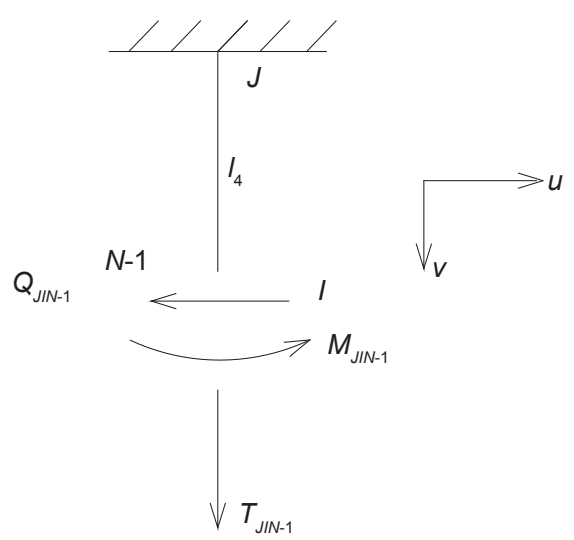

Fig. 3 Mechanical model of the straight part $J I$ 


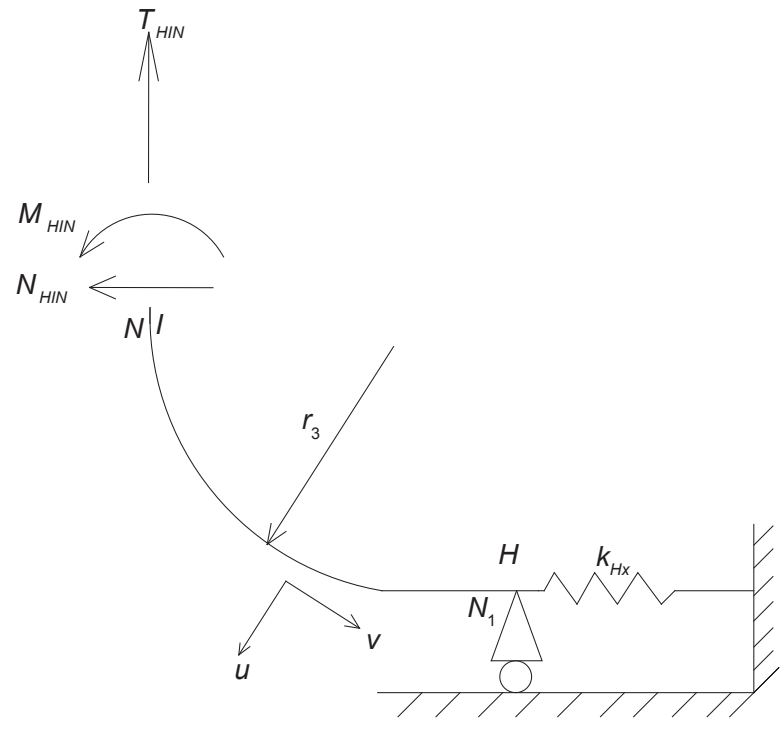

Fig. 4 Mechanical model of the curved part $I H$

\subsection{In-plane vibration of the part $J I$}

\subsubsection{Mechanical model of part $J I$ for coupling between} longitudinal and lateral vibrations

The straight part $J I$ can be simplified as a beam model as demonstrated in Fig. 5. For the coupling between longitudinal and lateral vibrations, the Hamilton equation of the beam model can be expressed as (Humar, 1990):

$$
\delta \int_{t 1}^{t 2}(T-U) \mathrm{d} t+\int_{t 1}^{t 2} \delta w \mathrm{~d} t=0
$$

where $T$ and $U$ are the kinetic energy and the potential energy of the beam; and $\delta w$ is the virtual work done by active forces.

For a point $B(x, y)$ in the beam and the other point $K(x, 0)$ in the beam axis, the displacements of point $B(x, y)$ are as follows:

$$
\left\{\begin{array}{l}
v_{B}=v+v_{B}^{\prime}=v-y u^{\prime} \\
u_{B}=u
\end{array}\right.
$$

where $u$ and $v$ are the displacements of point $K(x, 0)$. The stress and strain in the longitudinal direction at point $B(x, y)$ is then presented by:

$$
\left\{\begin{array}{l}
\varepsilon_{x}=\frac{\partial v_{B}}{\partial x}=-y u^{\prime \prime}+v^{\prime} \\
\sigma_{x}=E \varepsilon_{x}
\end{array}\right.
$$

where $E$ is Young's modulus.

The kinetic energy of the beam can be expressed as follows:

$$
\begin{aligned}
T & =\frac{1}{2} \iiint_{v} \rho\left(\dot{u}_{B}^{2}+\dot{v}_{B}^{2}\right) \mathrm{d} v \\
& =\frac{1}{2} \iiint_{v} \rho\left(y^{2} \dot{u}^{\prime 2}-2 y \dot{u}^{\prime} \dot{v}+\dot{u}^{2}+\dot{v}^{2}\right) \mathrm{d} v \\
& \approx \frac{1}{2} \int_{0}^{l} \rho A\left(\dot{u}^{2}+\dot{v}^{2}\right) \mathrm{d} x
\end{aligned}
$$

where $\rho$ is the density; $A$ is the sectional area.

The term $y^{2} \dot{u}^{\prime 2}$ is much smaller than $\dot{u}^{2}$ or $\dot{v}^{2}$, then it can be neglected. The integration of the term $y^{2} \dot{u}^{\prime 2}$ is 0 .

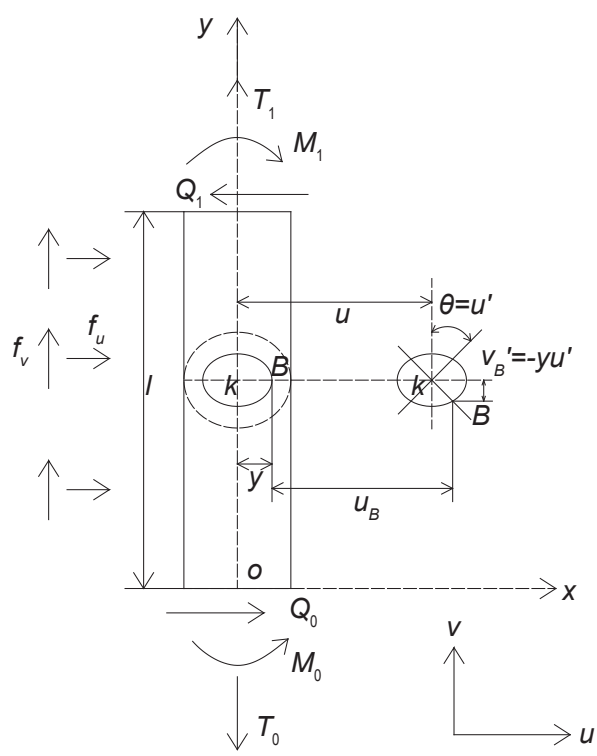

Fig. 5 Mechanical model of the part $J I$

The potential energy of the beam is:

$$
\begin{aligned}
U & =\frac{1}{2} \iiint_{v} \sigma_{x} \varepsilon_{x} \mathrm{~d} v \\
& =\frac{1}{2} \iiint_{v} E\left(y^{2} u^{\prime \prime 2}-2 y u^{\prime \prime} v^{\prime}+v^{\prime 2}\right) \mathrm{d} v \\
& =\frac{1}{2} \int_{0}^{l}\left(E I u^{\prime \prime 2}+E A v^{\prime 2}\right) \mathrm{d} x
\end{aligned}
$$

where $I$ is the moment of inertia. The integration of the term $-2 y u^{\prime \prime} v^{\prime}$ is 0 .

The virtual work created by active forces is:

$$
\begin{gathered}
\delta w=\int_{0}^{l}\left(f_{v} \delta v+f_{u} \delta u\right) \mathrm{d} x+Q_{0} \delta u_{0}-Q_{l} \delta u_{l} \\
-M_{0} \delta u_{0}^{\prime}+M_{l} \delta u_{l}^{\prime}-T_{0} \delta v_{0}+T_{l} \delta v_{l}
\end{gathered}
$$

where $f_{u}$ and $f_{v}$ are the active forces; $Q$ is the shearing force; and $T$ and $M$ stand for the axis force and moment respectively, as shown in Fig. 5.

Substituting Eqs. (4) through (7) into Eq. (1) gives:

$$
\begin{aligned}
& \int_{t 1}^{t 2} \int_{0}^{l}\left(-\rho A \ddot{v}+E A v^{\prime \prime}+f_{v}\right) \delta v \mathrm{~d} x \mathrm{~d} t \\
& +\int_{t 1}^{t 2} \int_{0}^{l}\left(-\rho A \ddot{u}-E I u^{\prime \prime \prime}+f_{u}\right) \delta u \mathrm{~d} x d t \\
& +\int_{t 1}^{t 2}\left(E I u_{l}^{\prime \prime \prime}-Q_{l}\right) \delta u_{l} \mathrm{~d} t+\int_{t 1}^{t 2}\left(-E I u_{0}^{\prime \prime \prime}+Q_{0}\right) \delta u_{0} \mathrm{~d} t \\
& +\int_{t 1}^{t 2}\left(-E I u_{l}^{\prime \prime}+M_{l}\right) \delta u_{l}^{\prime} \mathrm{d} t+\int_{t 1}^{t 2}\left(E I u_{0}^{\prime \prime}-M_{0}\right) \delta u_{0}^{\prime} \mathrm{d} t \\
& +\int_{t 1}^{t 2}\left(-E A v_{l}^{\prime}+T_{l}\right) \delta v_{l} \mathrm{~d} t+\int_{t 1}^{t 2}\left(E A v_{0}^{\prime}-T_{0}\right) \delta v_{0} \mathrm{~d} t \\
& =0
\end{aligned}
$$

From Eq. (7), we have:

$$
\left\{\begin{array}{l}
\rho A \ddot{v}-E F \frac{\partial^{2} v}{\partial x^{2}}-f_{v}=0 \\
\rho A \ddot{u}+E I \frac{\partial^{4} u}{\partial x^{4}}-f_{u}=0
\end{array}\right.
$$




$$
\left\{\begin{array}{l}
E I u_{0}^{\prime \prime \prime}=Q_{0} \\
E I u_{0}^{\prime \prime}=M_{0} \\
E A v_{0}^{\prime}=T_{0} \\
E I u_{l}^{\prime \prime \prime}=Q_{l} \\
E I u_{l}^{\prime \prime}=M_{l} \\
E A v_{l}^{\prime}=T_{l}
\end{array}\right.
$$

From Eq (8), it can be seen that the lateral vibration of the beam is decoupled from the longitudinal vibration when the small displacement is ignored.

\subsubsection{Dynamical model of the part $\boldsymbol{J I}$}

The dynamic equation of the part $J I$ can be obtained from Eq. (8):

$$
\left\{\begin{array}{l}
\rho A \ddot{u}_{J I}+E I \frac{\partial^{4} u_{J I}}{\partial x^{4}}-f_{u J I}=0 \\
\rho A \ddot{v}_{J I}-E F \frac{\partial^{2} v_{J I}}{\partial x^{2}}-f_{v J I}=0
\end{array}\right.
$$

The finite difference method is applied to solving Eq. (10). The beam is divided into $N$ elements, and the length of each element is $h=\frac{l_{4}}{N}$, we have:

$$
\left\{\begin{array}{l}
\ddot{u}_{J I k}+b_{1} u_{J I k+2}+b_{2} u_{J I k+1}+b_{3} u_{J I k}+b_{2} u_{J I k-1} \\
+b_{1} u_{J I k-2}+F_{u J I k}=0 \\
\ddot{v}_{J I k}+d_{2} v_{J I k+1}+d_{3} v_{J I k}+d_{2} v_{J I k-1}+F_{v J I k}=0
\end{array}\right.
$$

with

$$
\begin{aligned}
& b_{1}=\frac{E I}{\rho F h^{4}}, b_{2}=-4 b_{1}, b_{3}=6 b_{1} \\
& d_{1}=\frac{E}{\rho}, d_{2}=-\frac{d_{1}}{h^{2}}, d_{3}=-2 d_{2} \\
& F_{u J l k}=-\frac{f_{u J k}}{\rho A}, F_{v J I k}=-\frac{f_{v J k}}{\rho A}
\end{aligned}
$$

When $3 \leq k \leq N-3$, we obtain:

$$
\left(\begin{array}{cccccccccc}
b_{1} & 0 & b_{2} & 0 & b_{3} & 0 & b_{2} & 0 & b_{1} & 0 \\
0 & 0 & 0 & d_{2} & 0 & d_{3} & 0 & d_{2} & 0 & 0
\end{array}\right)\left(\begin{array}{l}
u_{J I k-2} \\
v_{J I k-2} \\
u_{J I k-1} \\
v_{J I k-1} \\
u_{J I k} \\
v_{J I k} \\
u_{J I k+1} \\
v_{J I k+1} \\
u_{J I k+2} \\
v_{J I k+2}
\end{array}\right)+\left(\begin{array}{l}
\ddot{u}_{J I k} \\
\ddot{v}_{J l k}
\end{array}\right)=\left(\begin{array}{l}
-F_{u J l k} \\
-F_{v J l k}
\end{array}\right)
$$

By considering the boundary conditions $u_{J I 0}=0$, $v_{J I 0}=0, \alpha_{0}=\partial u_{J I 0} / \partial x=0$ we have:

$$
u_{J I 0}=0, v_{J I 0}=0, u_{J I 1}=u_{J I-1}
$$

When $K=1$, 2, we have:

$$
\left(\begin{array}{l}
\ddot{u}_{J I 1} \\
\ddot{v}_{J I 1} \\
\ddot{u}_{J I 2} \\
\ddot{v}_{J I 2}
\end{array}\right)+\left(\begin{array}{cccccccc}
b_{4} & 0 & b_{2} & 0 & b_{1} & 0 & 0 & 0 \\
0 & d_{3} & 0 & d_{2} & 0 & 0 & 0 & 0 \\
b_{2} & 0 & b_{3} & 0 & 0 & b_{1} & 0 & 0 \\
0 & d_{2} & 0 & d_{3} & 0 & d_{2} & 0 & 0
\end{array}\right)\left(\begin{array}{l}
u_{J I 1} \\
v_{J I 1} \\
u_{J I 2} \\
v_{J I 2} \\
u_{J I 3} \\
v_{J I 3} \\
u_{J I 4} \\
v_{J I 4}
\end{array}\right)=\left(\begin{array}{l}
-F_{u J I 1} \\
-F_{v J I 1} \\
-F_{u J I 2} \\
-F_{v J I 2}
\end{array}\right)
$$$$
\text { where } b_{4}=b_{1}+b_{3} \text {. }
$$

\subsection{In-plane vibration of the part $\mathbf{I H}$}

For spools of subsea pipelines, Yue (2004) did comprehensive research on the in-plane vibration. He deduced a dynamical equation by the analytical method for the coupling between the longitudinal and lateral vibrations of the curved part $I H$. The Newmark Integration Method (Newmark, 1959) was used for the solution of the developed equations. As shown in Fig. 4, the part $I H$ is divided into $N_{1}$ elements, and the angle of each element is $\theta_{1}=\frac{\pi}{2 N_{1}}$. The
boundary conditions are as follows:

$T_{I H N_{1}}=-k_{H x} v_{I H N_{1}}, Q_{I H N_{1}}=k_{H z} u_{I H N_{1}}, M_{I H N_{1}}=-k_{H \theta} \alpha_{I H N_{1}}$

where $k_{H x}, k_{H z}$, and $k_{H \theta}$ are equivalent spring coefficients.

And when $K=N_{1}+1$, an additional condition can be expressed as the following form:

$$
u_{I H N_{1}+1}=-\frac{\partial v_{I H N_{1}+1}}{\partial \theta}
$$

The combination of Eq. (15) and Eq. (16), for $2 \leq k \leq N_{1}$, gives:

$$
\begin{aligned}
& \left(\begin{array}{cccccccccc}
\ddots & & & & & & & \\
c_{4} & -c_{23} & c_{21} & -c_{22} & c_{20} & 0 & c_{21} & c_{22} & c_{4} & c_{23} \\
-c_{8} & 0 & -c_{24} & c_{26} & 0 & c_{25} & c_{24} & c_{26} & c_{8} & 0 \\
\vdots & & & & & & & \\
0 & 0 & a_{119} & a_{120} & a_{121} & a_{122} & a_{123} & a_{124} & a_{125} & a_{126} \\
0 & 0 & a_{127} & a_{128} & a_{129} & a_{130} & a_{131} & a_{132} & a_{133} & a_{134} \\
0 & 0 & 0 & 0 & a_{100} & a_{101} & a_{102} & a_{103} & a_{104} & a_{105} \\
0 & 0 & 0 & 0 & a_{106} & a_{107} & a_{108} & a_{109} & a_{110} & a_{111}
\end{array}\right)\left(\begin{array}{c}
\vdots \\
u_{I H k} \\
v_{I H k} \\
\vdots \\
u_{I H N_{1}-1} \\
\ddot{u}_{I H k} \\
\ddot{v}_{I H k} \\
\vdots \\
\ddot{u}_{I H N_{1}-1} \\
\ddot{v}_{I H N_{1}-1} \\
\ddot{u}_{I H N_{1}} \\
\ddot{v}_{I H N_{1}}
\end{array}\right)=\left(\begin{array}{c}
-F_{u I H k} \\
-F_{v I H k} \\
\vdots \\
-F_{u I H N_{1}-1} \\
-F_{v I H N_{1}-1} \\
-F_{u I H N_{1}} \\
-F_{v I H N_{1}}
\end{array}\right) \\
& \left(\begin{array}{c}
v_{I H N_{1}}
\end{array}\right)
\end{aligned}
$$

where the above parameters are defined by Yue (2004). 


\subsection{Derivation of the dynamic equations for the part $\mathbf{J H}$}

The boundary conditions at the point $I$ should be satisfied:

$$
\left\{\begin{array}{l}
u_{J I N}=-u_{I H 0}, v_{J N}=v_{I H 0}, \theta_{J N}=-\theta_{I H 0} \\
T_{J I N-1}=T_{I H 0}, M_{J I N-1}=-M_{I H 0}, Q_{J I N-1}=-Q_{I H 0}
\end{array}\right.
$$

For the curved part $I H$, when $k=-1$ we have:

$$
u_{I H-1}=-\frac{\partial v_{I H-1}}{\partial \theta}
$$

From Eqs. (18) and (19), the following equations are obtained with the finite difference method:

$$
\left\{\begin{array}{l}
u_{J I N}=-u_{I H 0} \\
v_{J I N}=v_{I H 0} \\
b_{5} u_{J I N+1}-b_{5} u_{J I N-1}+u_{I H 1}-u_{I H-1}+b_{10} v_{I H 0}=0 \\
b_{5} v_{J I N}-b_{5} v_{J I N-2}-v_{I H 1}+v_{I H-1}+b_{10} u_{I H 0}=0 \\
b_{8} u_{J I N}-2 b_{8} u_{J I N-1}+b_{8} u_{J I N-2}+b_{6} u_{I H 1}-2 b_{6} u_{I H 0}+b_{6} u_{I H-1}+b_{7} v_{I H 1}-b_{7} v_{I H-1}=0 \\
b_{13} u_{J I N+1}-2 b_{13} u_{J I N}+2 b_{13} u_{J I N-2}-b_{13} u_{J I N-3}+b_{11} u_{I H 2}-2 b_{11} u_{I H 1}+2 b_{11} u_{I H-1}-b_{11} u_{I H-2}-b_{12} v_{I H 1}+2 b_{12} v_{I H 0}-b_{12} v_{I H-1}=0 \\
v_{I H 0}-v_{I H-2}-b_{10} u_{I H-1}=0
\end{array}\right.
$$

which gives:

$$
\left\{\begin{array}{l}
u_{J I N}=-u_{I H 0} \\
v_{J I N}=v_{I H 0} \\
u_{J I N+1}=d_{10} u_{I H 2}+d_{11} u_{I H 1}+d_{12} u_{I H 0}+d_{13} v_{I H 1}+d_{14} v_{I H 0}+d_{15} u_{J I N-3}+d_{16} u_{J I N-2}+d_{17} u_{J N-1}+d_{18} v_{J I N-2}+d_{19} v_{J I N-1} \\
u_{I H-2}=d_{20} u_{I H 2}+d_{21} u_{I H 1}+d_{22} u_{I H 0}+d_{23} v_{I H 1}+d_{24} v_{I H 0}+d_{25} u_{J I N-3}+d_{26} u_{J N-2}+d_{27} u_{J I N-1}+d_{28} v_{J I N-2}+d_{29} v_{J I N-1} \\
v_{I H-2}=d_{30} u_{I H 2}+d_{31} u_{I H 1}+d_{32} u_{I H 0}+d_{33} v_{I H 1}+d_{34} v_{I H 0}+d_{35} u_{J I N-3}+d_{36} u_{J I N-2}+d_{37} u_{J I N-1}+d_{38} v_{J I N-2}+d_{39} v_{J N-1} \\
u_{I H-1}=d_{40} u_{I H 2}+d_{41} u_{I H 1}+d_{42} u_{I H 0}+d_{43} v_{I H 1}+d_{44} v_{I H 0}+d_{45} u_{J I N-3}+d_{46} u_{J I N-2}+d_{47} u_{J I N-1}+d_{48} v_{J I N-2}+d_{49} v_{J I N-1} \\
v_{I H-1}=d_{50} u_{I H 2}+d_{51} u_{I H 1}+d_{52} u_{I H 0}+d_{53} v_{I H 1}+d_{54} v_{I H 0}+d_{55} u_{J I N-3}+d_{56} u_{J I N-2}+d_{57} u_{J I N-1}+d_{58} v_{J I N-2}+d_{59} v_{J I N-1}
\end{array}\right.
$$

For the part $J I$ when $k=N-2, N-1$, and the part $I H$ when $k=0,1$, Eq. (21) yields:

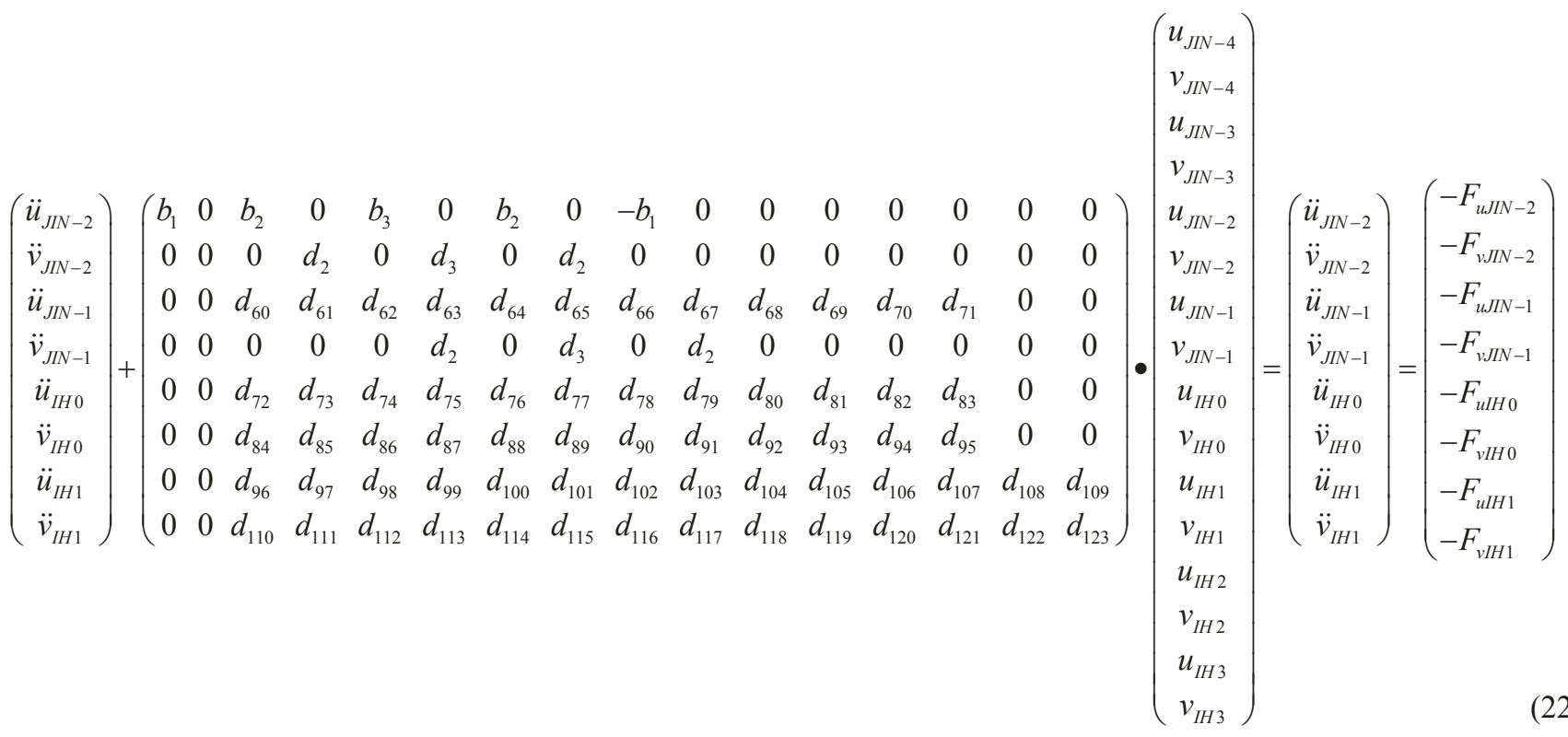


where the above parameters are defined by Yue (2004).

The in-plane vibration of the riser $J H$ is deduced from Eqs. (12), (14), (17) and (22):

$$
M \ddot{U}+K U=F
$$

or if the influence of Rayleigh damping is included:

$$
M \ddot{U}+C \dot{U}+K U=F
$$

where the damping matrix $C=\alpha M+\beta K, 2 \xi_{i} \omega_{i}=\alpha+\beta \omega_{i}^{2}$, and $\alpha$ is a mass damping coefficient and $\beta$ a rigidity damping coefficient. In practice, $\alpha=0, \beta=2 \xi_{i} / \omega_{i}$, For steels, the damping ratio $\xi_{i}=0.02$, and the first frequency of the riser can be excited easily by earthquake, which yields $\beta=0.04 / \omega_{1}$.

\section{Numerical results and discussion}

For a riser in Bohai Bay, China, the parameters are detailed in Table 1 where $S_{4}=0$ indicates that the point $H$ and $G_{1}$ are the same point, and the point $H$ is fixed, and $k_{H x}=\infty$.

The Tianjin Infrequent Wave (TIW) and the Elcentro Earthquake Wave (EEW) are respectively applied to the seismic response analysis of the riser. Figs. 6 and 7 present respectively the acceleration curves of the Tianjin Infrequent Wave and the Elcentro Earthquake Wave.

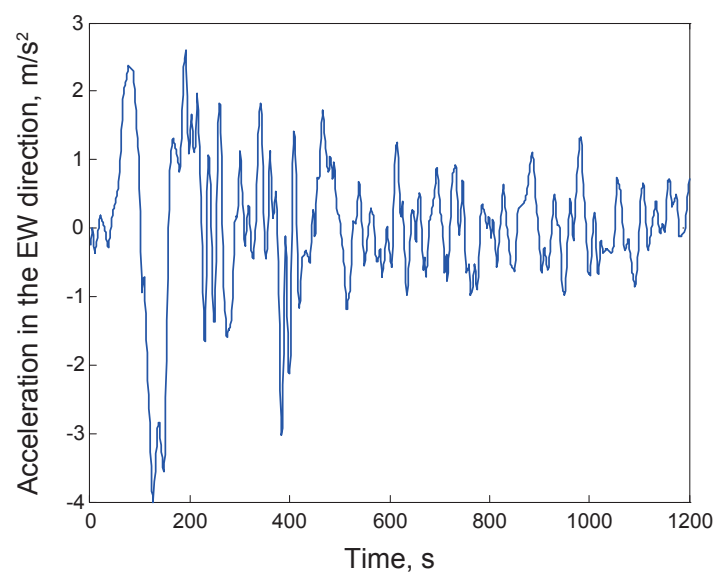

(a) EW direction

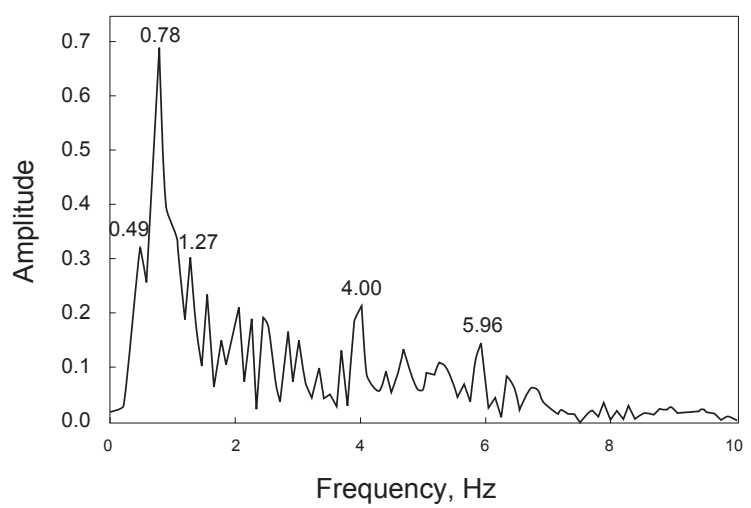

(c) Spectrum in the EW direction
Table 1 The parameters of a typical riser in Bohai Bay, China (CNOOCRC, 2002)

\begin{tabular}{cc}
\hline Parameter & Value \\
\hline Outside diameter $D, \mathrm{~m}$ & 0.2191 \\
Inside diameter $d, \mathrm{~m}$ & 0.19971 \\
Young's modulus $E, \mathrm{~Pa}$ & $2.03 \mathrm{e} 11$ \\
Density $\rho, \mathrm{kg} / \mathrm{m}^{3}$ & $7.8 \mathrm{e} 3$ \\
Length of the straight part $l_{4}, \mathrm{~m}$ & 100 \\
Length of the straight part $S_{3}, \mathrm{~m}$ & 0 \\
Radius of the curved part $r_{3}, \mathrm{~m}$ & 1.5955 \\
\hline
\end{tabular}

The fundamental frequency of the riser can be estimated simply (Yue et al, 2006):

$$
f=\frac{4.73^{2}}{2 \pi l^{2}} \sqrt{\frac{E I}{\rho F}}
$$

with

$$
l=l_{4}+\frac{\pi}{2} r_{3}
$$

The fundamental frequency of $0.13 \mathrm{~Hz}$ of the riser system is obtained from Eq. (25) and Table 1. By taking coefficients $\alpha=0, \beta=0.04 / \omega_{1}=0.049$, the maximum stresses are calculated (listed in Table 2), and the stress responses of the riser due to TIW in the EW direction are shown in Fig. 8.

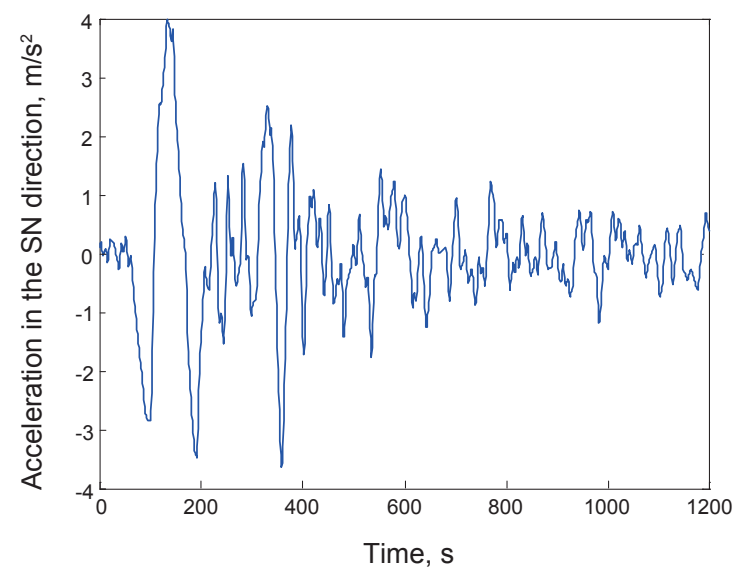

(b) $\mathrm{SN}$ direction

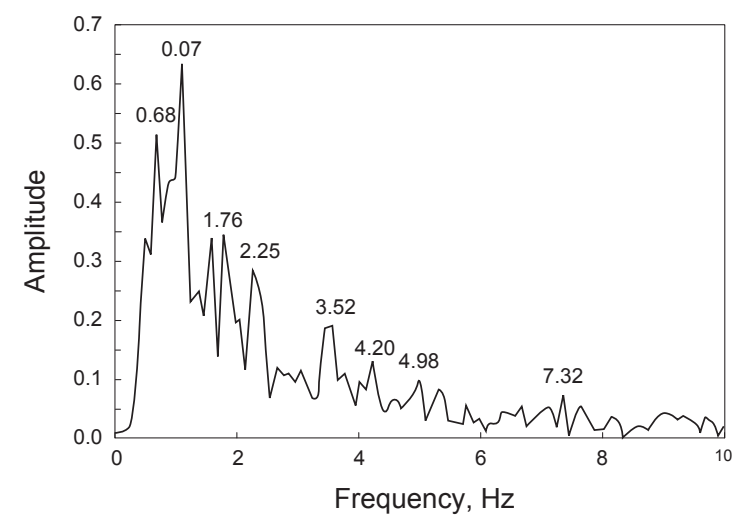

(d) Spectrum in the $\mathrm{SN}$ direction

Fig. 6 Tianjin Infrequent Wave 
Table 2 and Fig. 8 show that the maximum stress of the riser is the normal stress due to the bending moment.

Currently, the offshore oilfields in China are located in water depth of $5-330 \mathrm{~m}$. Table 3 presents the calculated results of the maximum seismic stresses for different riser length $l_{4}$.

The dependence of the maximum stress in the riser on the length of $l_{4}$ is illustrated in Table 3 , showing higher values in the range of $30 \mathrm{~m}<l_{4}<100 \mathrm{~m}$. Engineering practice requires that the earthquake influence be included if the maximum seismic stress in the subsea pipelines exceeds $30 \mathrm{MPa}$. Therefore, seismic stresses of the risers for most platforms must be presented in active earthquake zones for design checking.

Further analysis of Figs. 6 and 7 prove the dependence of the seismic stress on the riser length as shown in Table 3. The amplitude or energy of the TIW is focused in a very small range within $2 \mathrm{~Hz}$, while the EEW within $4 \mathrm{~Hz}$. The maximum amplitude of the TIW and the corresponding maximum stress of the riser are focused in the same range between $0.49 \mathrm{~Hz}$ and $1.27 \mathrm{~Hz}$. It is apparent that an increase in the riser length decreases the fundamental frequency of the structure, and resonance of the system will occur when the frequency falls in the range of the frequencies of earthquake wave, presenting higher seismic stresses in the riser. Shorter risers induce smaller seismic stresses due to their larger

Table 2 The maximum stress of the riser induced by earthquake waves

\begin{tabular}{ccccc}
\hline $\begin{array}{c}\text { Earthquake waves } \\
\text { in different directions }\end{array}$ & $\begin{array}{c}\text { Maximum normal } \\
\text { stress of the part } J I\end{array}$ & $\begin{array}{c}\text { Maximum shear } \\
\text { stress of the part } J I\end{array}$ & $\begin{array}{c}\text { Maximum normal } \\
\text { stress of the part } I H\end{array}$ & $\begin{array}{c}\text { Maximum shear stress } \\
\text { of the part } I H\end{array}$ \\
\hline TIW in the EW direction & 50.3 & 0.356 & 48.4 & 2.07 \\
TIW in the SN direction & 44.8 & 0.333 & 43.4 & 1.89 \\
EEW in the EW direction & 88.0 & 0.327 & 86.2 & 3.37 \\
EEW in the SN direction & 24.1 & 0.191 & 23.5 & 0.926 \\
\hline
\end{tabular}

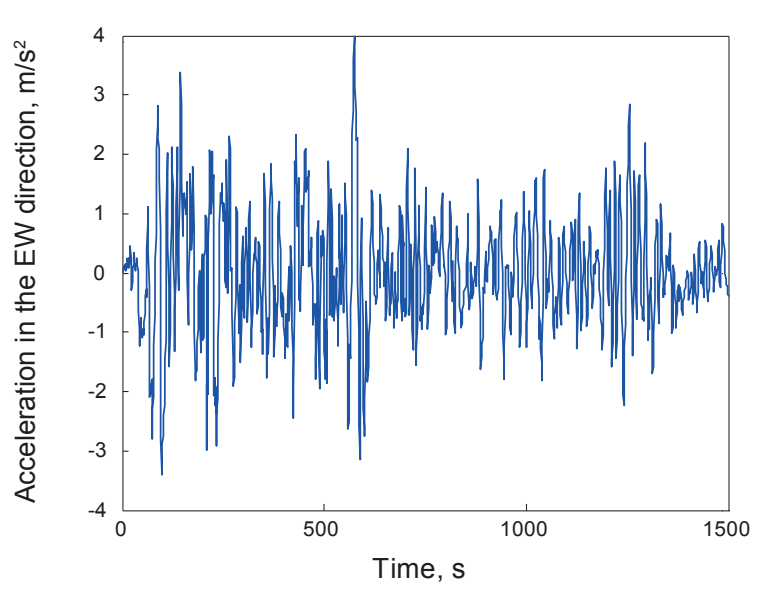

(a) EW direction

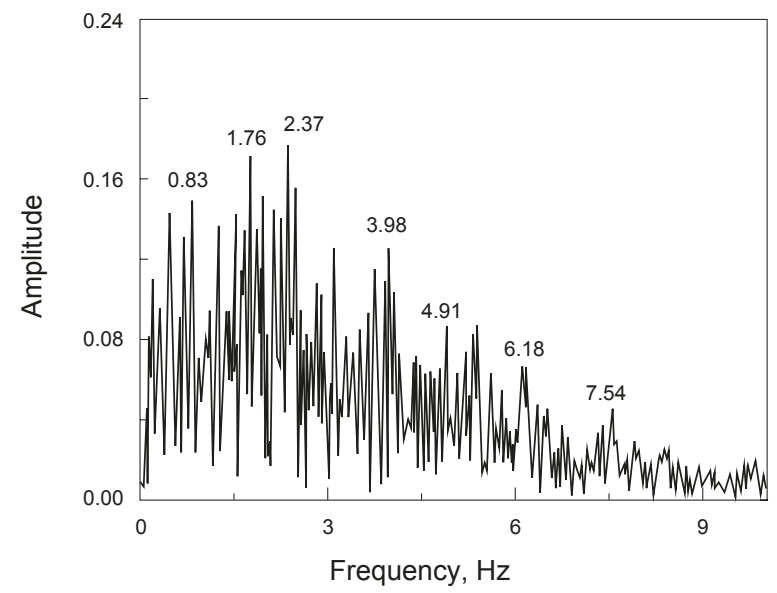

(c) Spectrum in the EW direction

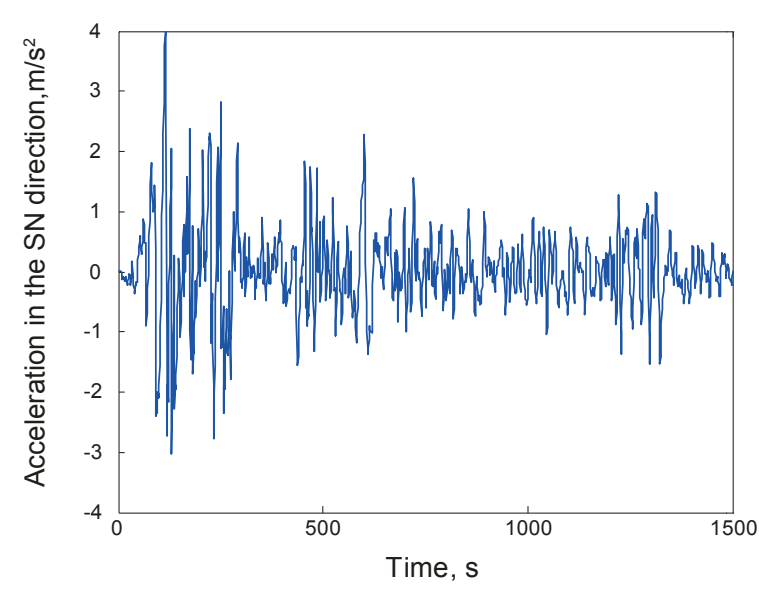

(b) SN direction

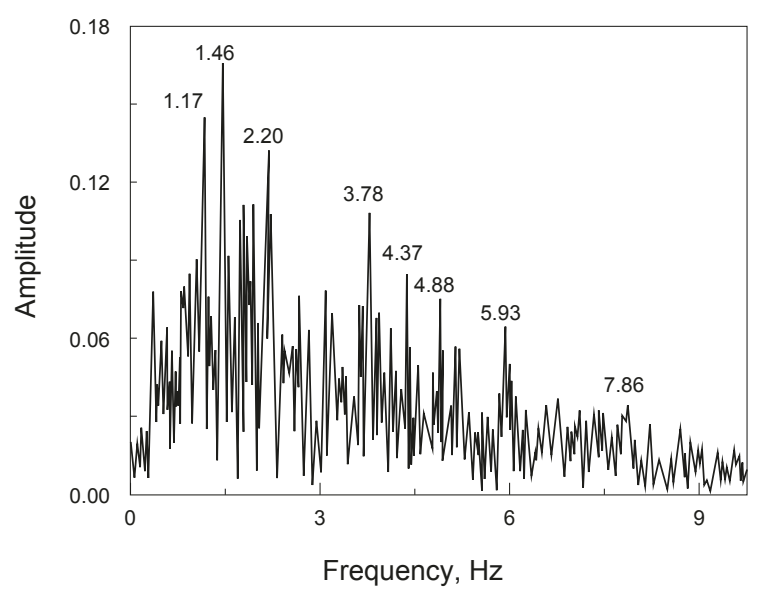

(d) Spectrum in the $\mathrm{SN}$ direction

Fig. 7 Elcentro Earthquake Wave 


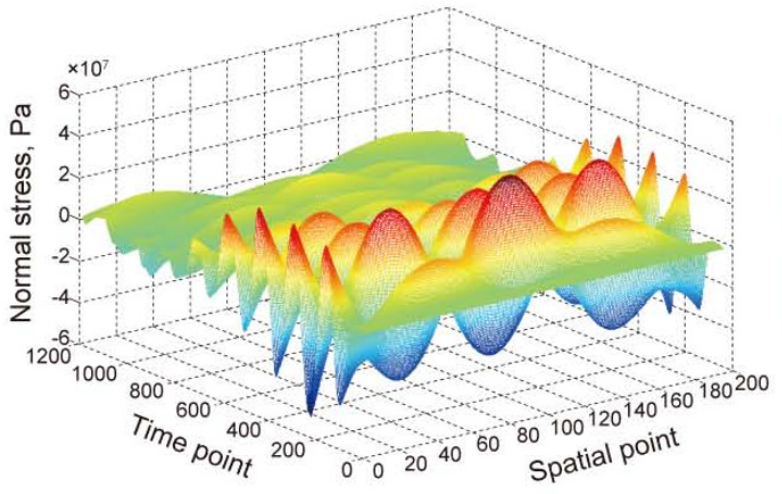

(a) Normal stress due to bending moment in the part $\mathrm{Jl}$

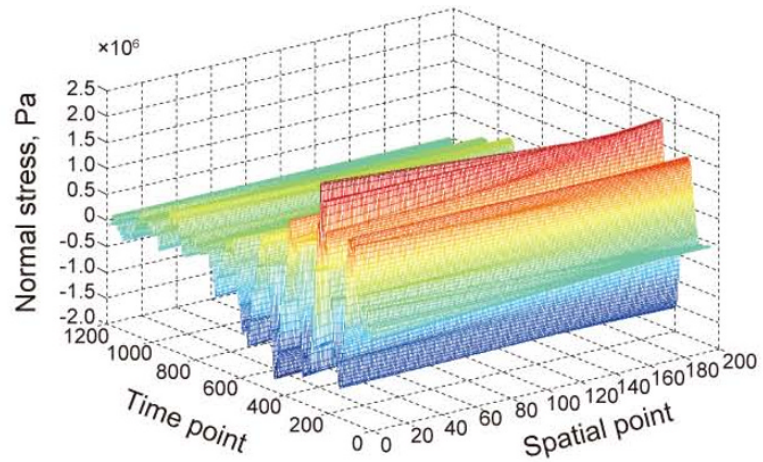

(c) Normal stress due to axial force in the part $\mathrm{Jl}$

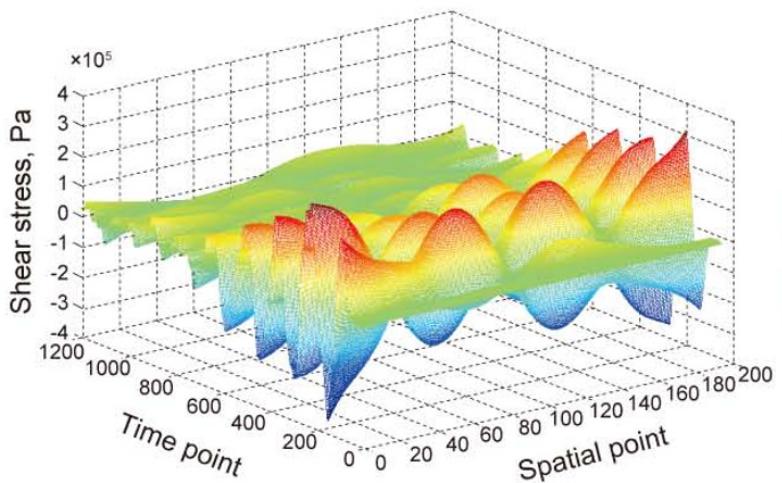

(e) Shear stress due to shear force in the part $\mathrm{Jl}$

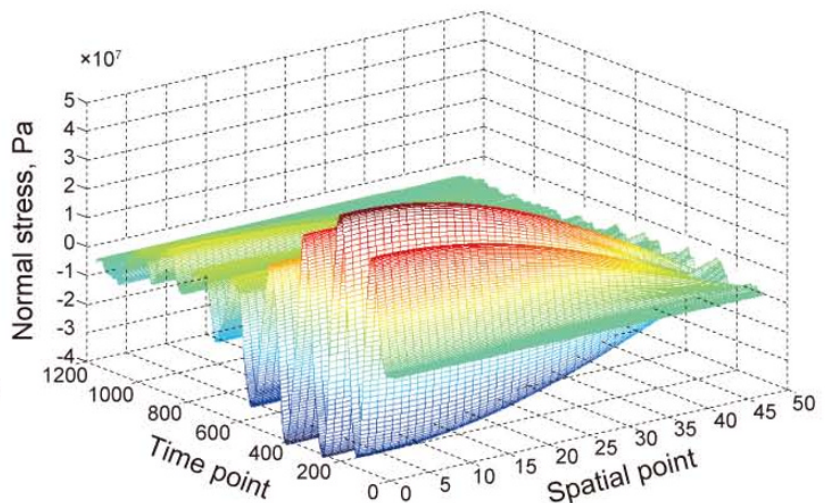

(b) Normal stress due to bending moment in the part IH

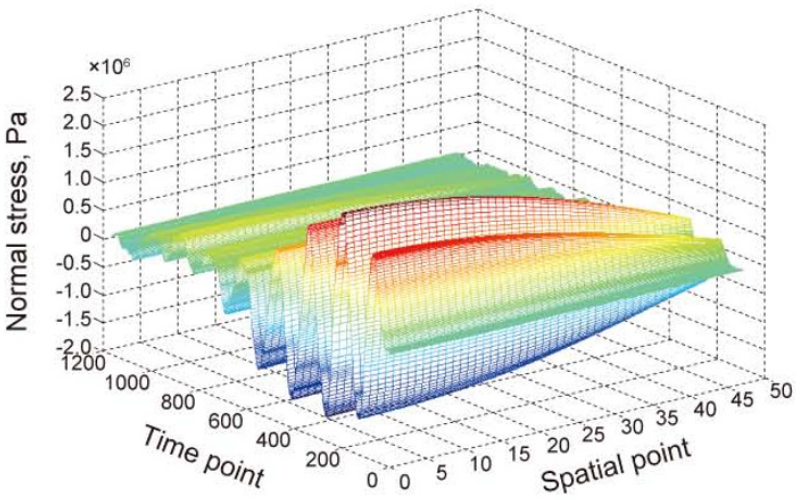

(d) Normal stress due to axial force in the part $I H$

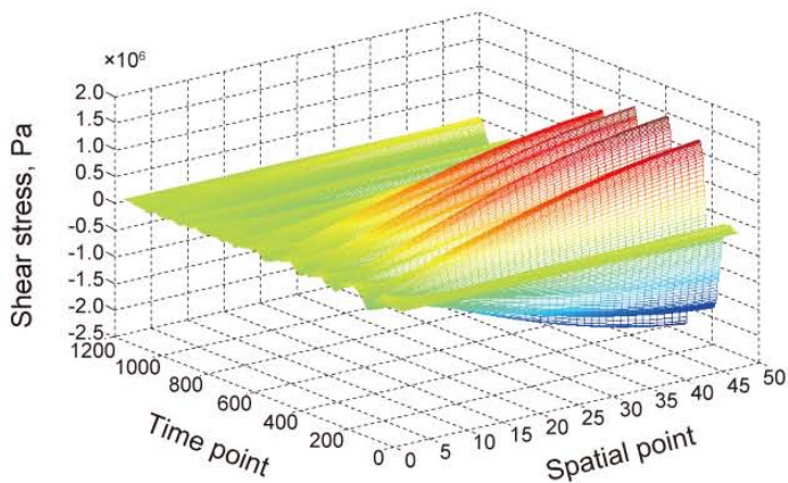

(f) Shear stress due to shear force in the part $I H$

Fig. 8 Stress responses of the riser induced by the TIW in the EW direction

fundamental frequencies which are far away from frequencies of earthquake waves. Fixing the risers to the platform legs by installing clamps is an effective measure to increase the fundamental frequency of the riser system, which is also in agreement with the measures for reducing the vortex induced vibration of the risers. It is recommended that the fundamental frequency of the riser be higher than $4 \mathrm{~Hz}$ in preliminary design of the system, and Eq. (25) is a basic presentation for calculating the riser frequency. For detailed design checking against earthquake waves, methods developed in this paper are strongly recommended.

\section{Conclusions}

Risers form an important part of subsea pipelines, and no criterion for seismic design for risers is available. A dynamic model for seismic analysis of risers is developed in this paper. The dynamic equations are derived from the Hamilton theory and the seismic responses of the riser system are obtained on the basis of numerical calculation and analysis using the Newmark Integration Method. The following conclusions can be made:

1) The maximum stress of the riser is the normal stress. 
Table 3 A comparison of the maximum stresses

\begin{tabular}{|c|c|c|c|c|c|}
\hline \multirow{2}{*}{$\begin{array}{c}\text { Length of the } \\
\text { straight part } l_{4}, \mathrm{~m}\end{array}$} & \multirow{2}{*}{$\begin{array}{l}\text { Fundamental } \\
\text { frequency, } \mathrm{Hz}\end{array}$} & \multicolumn{4}{|c|}{ The maximum stress, $\mathrm{MPa}$} \\
\hline & & TIW, EW direction & TIW, SN direction & EEW, EW direction & EEW, SN direction \\
\hline 10 & 8.61 & 6.32 & 7.02 & 6.88 & 7.21 \\
\hline 20 & 2.66 & 38.7 & 34.9 & 48.0 & 41.2 \\
\hline 30 & 1.27 & 90.6 & 112 & 99.9 & 87.0 \\
\hline 40 & 0.75 & 213 & 189 & 149 & 70.1 \\
\hline 50 & 0.49 & 132 & 138 & 143 & 73.5 \\
\hline 60 & 0.34 & 78.5 & 60.9 & 140 & 90.0 \\
\hline 80 & 0.20 & 52.2 & 46.9 & 89.8 & 24.6 \\
\hline 100 & 0.13 & 50.3 & 44.8 & 88.0 & 24.1 \\
\hline 150 & 0.058 & 34.8 & 32.9 & 52.8 & 30.2 \\
\hline 200 & 0.033 & 31.6 & 29.2 & 49.6 & 20.1 \\
\hline 300 & 0.015 & 25.5 & 22.7 & 37.7 & 33.7 \\
\hline
\end{tabular}

2) The maximum stress in the riser strongly depends on the length of the riser, and seismic responses must be provided for riser design in active earthquake zones.

3) The fundamental frequency of the riser system is the main parameter controlling the seismic responses, and resonance occurs when the frequency falls in the range of the frequencies of earthquake waves which will induce higher seismic stresses in the riser. In this case, methods developed in this paper are strongly recommended for detailed design checking.

4) Eq. (25) is a simple solution for preliminary design of the risers against earthquake waves, making sure that the fundamental frequency of the riser be higher than $4 \mathrm{~Hz}$. If not, detailed analysis of seismic responses by applying the methods developed in this paper is recommended to perform.

5) The Tianjin Infrequent Wave and the Elcentro Wave are used only for numerical analysis in this paper. For engineering design of risers, in-situ records of earthquake waves are strongly recommended for more practical calculations.

\section{Acknowledgements}

This research is financially supported by the National Natural Science Foundation of China (granted number 50979113), and funded by the National 863 Program of China (granted number 2006AA09A105). Thanks also go to Prof. Decheng Chen from Peking University for his critical reading of the manuscript.

\section{References}

Bruschi R, Curti G, Marchesani F, et al. Upgrades for deep water pipelines on very uneven seabeds. Deep Offshore Technology Conference. 1993. 98-140

Bruschi R, Marchsani F, Vitali L, et al. Structural integrity of offshore pipelines in seismic conditions. International Conference on Offshore Mechanics and Arctic Engineering. Florence, Italy. 1996. 5: 421-429 China Classification Society (CCS). Rules for Submarine Pipeline
Systems. Beijing: China Communications Press. 1992 (in Chinese) China National Offshore Oil Research Center. QHD32 Project ODP Report. CNOOC Research Center, Engineering Report. Beijing. 2002 (in Chinese)

Det Norske Veritas (DNV). Submarine Pipeline System. DNV-OS-F101. Hovik, Norway. 2000

Det Norske Veritas (DNV). Dynamic risers. DNV-OS-F201. Hovik, Norway. 2001

Duan M L and Sun Z C. Ultimate stress analysis of subsea pipeline design against earthquakes. International Offshore and Polar Engineering Conference. Toulon, France. May 23-28, 2004. 2: 77-81

Humar J L. Dynamics of Structures. New Jersey: Prentice-Hall. 1990. 632-638

Kershenbaum N Y, Mebarkia S A and Choi H S. Behavior of marine pipelines under seismic faults. Ocean Engineering. 2000. 27 (5): 473-483

Matsubara K and Hoshiya M. Soil spring constants of buried pipelines for seismic design. Journal of Engineering Mechanics-ASCE. 2000. 126: $76-83$

Newmark N M. A method of computation for structural dynamics. Journal of Engineering Mechanics-ASCE. 1959. 85: 67-94.

Ogawa Y and Koike T. Structural design of buried pipelines for severe earthquakes. Soil Dynamics and Earthquake Engineering. 2001. 21: 199-209

Paulin M L, Phillips R, Clark J I, et al. Establishment of a full-scale pipeline/soil interaction test facility and results from lateral and axial investigations in sand. Offshore Mechanics and Arctic Engineering Conference. Yokohama. 1997. 5: 139-146

Romagnoli R and Varvelli R. An integrated seismic response analysis of offshore pipeline-sea floor systems. Offshore Mechanics and Arctic Engineering Conference. Houston, Texas. 1988. 139-145

Sun Z C, Duan M L, Liu L M, etc. The analyzing methods and advice on seismic stress calculation of offshore pipelines. China Engineering Sciences. 2003. 5(8): 76-80 (in Chinese)

Sun Z C, Duan M L and Zhang W. Some problem and solution of seismic design for subsea pipeline. Journal of Vibration Engineering. 2004. 17(3): 263-268 (in Chinese)

Sun Z C, Duan M L and Zhang W. Seismic design methods for submarine pipelines resistant against earthquake waves. Acta Petrolei Sinica. 2005. 26(2): 115-118 (in Chinese) 
Sun Z C, Duan M L, Zhang W, et al. Method for ultimate stress in seismic design of subsea pipelines. Journal of Applied Mechanics. 2006. 23(3): 436-440 (in Chinese)

Tura F, Dumitrescu A, Bryndum M B, et al. Guidelines for free spanning pipelines: the GUDESP project. Offshore Mechanics and Arctic Engineering Conference. Houston, Texas. 1994. 247-256

Xie B, Duan M L and Qing T Y. Reliability evaluation for fatigue and fracture of riser in deep offshore. Acta Petrolei Sinica. 2004. 25(3):
95-100 (in Chinese)

Yue Z Y. The Study of the Seismic Design of Subsea Pipelines and Buckling of Pipelines with Initial Imperfections. Ph.D Thesis. Peking University. Beijing, China. 2004 (in Chinese)

Yue Z Y, Duan M L and Sun Z C. Seismic design of risers. Journal of Vibration Engineering. 2006. 19(S): 23-27 (in Chinese)

(Edited by Sun Yanhua) 